\title{
Detection of magnetic fields in He-rich early B-type stars using HARPSpol
}

\author{
S. P. Järvinen ${ }^{1}$, S. Hubrig ${ }^{1}$, I. Ilyin ${ }^{1}$, M. Schöller ${ }^{2}$, M. F. Nieva ${ }^{3}$, N. Przybilla ${ }^{3}$, and N. Castro ${ }^{4}$ \\ 1 Leibniz-Institut für Astrophysik Potsdam (AIP), An der Sternwarte 16, 14482 Potsdam, Germany \\ e-mail: sjarvinen@aip.de \\ 2 European Southern Observatory, Karl-Schwarzschild-Str. 2, 85748 Garching, Germany \\ 3 Institut für Astro- und Teilchenphysik, Universität Innsbruck, Technikerstr. 25/8, 6020 Innsbruck, Austria \\ 4 Department of Astronomy, University of Michigan, 1085 S. University Avenue, Ann Arbor, MI 48109-1107, USA
}

Received 6 April 2018 / Accepted 6 August 2018

\begin{abstract}
Aims. We focus on early-B type stars with helium overabundance, for which the presence of a magnetic field has not previously been reported.

Methods. The measurements were carried out using high-spectral-resolution spectropolarimetric observations obtained with the High Accuracy Radial velocity Planet Searcher (HARPS) in polarimetric mode, installed at the ESO La Silla $3.6 \mathrm{~m}$ telescope.

Results. For five He-rich stars, the longitudinal magnetic field was detected for the first time. For one target, HD 58260, the presence of a longitudinal magnetic field of the order of $1.8 \mathrm{kG}$ has already been reported in the literature, but the magnetic field has remained constant over tens of years. Our measurement carried out using the polarimetric spectra obtained in 2015 March indicates a slight decrease of the longitudinal magnetic field strength compared to measurements reported in previous works. A search for periodic modulation in available photometric data allowed us to confidently establish a period of $2.64119 \pm 0.00420 \mathrm{~d}$ in archival ASAS3 data for CPD $-27^{\circ} 1791$. No period could be determined for the other five stars.

Conclusions. The obtained results support the scenario that all He-rich stars are detectably magnetic and form an extension of the Ap star phenomenon to higher temperatures.
\end{abstract}

Key words. stars: chemically peculiar - stars: early-type - stars: magnetic field

\section{Introduction}

During the last years an increasing number of massive stars has been investigated for magnetic fields in the framework of the Magnetism in Massive Stars (MiMeS) and B-fields in OB stars (BOB) surveys (Wade et al. 2016; Morel et al. 2015). Direct magnetic-field measurements are of great importance to properly understand the potential effects of magnetic fields on the evolution of massive stars, including the impact on angular momentum and on stellar wind properties. While the BOB survey mostly concentrated on normal main sequence OB stars, the MiMeS survey consisted of a survey component and a targeted component to characterise a sample of known magnetic stars. Since previously detected magnetic $\mathrm{O}$ and early B-type stars on average appeared to have rotation velocities significantly lower than the rest of the population, to enhance the probability of detecting magnetic fields, the majority of the stars targeted by BOB were relatively slowly rotating, with $v \sin i$ values below $60 \mathrm{~km} \mathrm{~s}^{-1}$ (Morel et al. 2015). However, according to Schöller et al. (2017), the results of the search for the presence of a magnetic field in these stars did not result in higher yields, with a magnetic field detection rate of $5 \pm 5 \%$.

While these results indicate that the presence of a magnetic field in normal, slowly rotating $\mathrm{O}$ and early B-type stars is not common, magnetic early B-type He-rich stars constitute about $10 \%$ of all main sequence early-B type stars. This group contains the most massive chemically peculiar stars with spectral types around B2 and exhibits helium and silicon surface spots (e.g. Landstreet \& Borra 1978; Borra \& Landstreet 1979; Bohlender 1988; Hubrig et al. 2017; Castro et al. 2017). The distribution of these spots is non-uniform and non-symmetric with respect to the rotation axis. Also, lines belonging to $\mathrm{CNO}$ and iron peak elements frequently show variable line profiles over the rotation periods, but the distribution of these elements is poorly documented in the literature due to the relative weakness of their lines compared to $\mathrm{He}$ and $\mathrm{Si}$ lines.

From previous studies of He-rich stars, we know that inhomogeneous chemical abundance distributions in early-B type stars are only observed on the surface of magnetic chemically peculiar stars with large-scale organised magnetic fields. Among such stars, five stars, CPD-27 1791, HD 60344, HD 149257, CPD-69 2698 , and HD 168785 , have previously been reported to exhibit a surface helium overabundance (e.g. MacConnell et al. 1970; Garrison et al. 1977). As no definite magnetic fields were reported in previous studies of these stars, they were included in the BOB target list. Obviously, these He-rich stars are excellent candidates to confirm the positive correlation between the presence of a magnetic field and helium enrichment in the star's atmosphere. The sixth star in our sample, HD 58260, was shown to be magnetic by Borra \& Landstreet (1979), but exhibited a surprisingly constant magnetic field over nine years (Bohlender et al. 1987). A recent study of this star by 
Table 1. Logbook of the observations.

\begin{tabular}{lccc}
\hline \hline Star & $m_{\mathrm{V}}$ & $\begin{array}{c}\text { HJD } \\
2400000+\end{array}$ & $S / N$ \\
\hline CPD-27 1791 & 9.3 & 57095.6063 & 162 \\
HD 58260 & 6.7 & 57091.6462 & 329 \\
HD 60344 & 9.1 & 57093.6454 & 258 \\
HD 149257 & 9.2 & 57095.7374 & 234 \\
CPD-69 $2698^{\circ}$ & 9.3 & 57178.6338 & 154 \\
HD 168785 & 8.5 & 57095.8469 & 265 \\
HD 168785 & 8.5 & 57316.5335 & 209 \\
\hline
\end{tabular}

Notes. The columns give the name of the star, the visual magnitude, the heliocentric Julian date (HJD) for the middle of the exposures, and the $\mathrm{S} / \mathrm{N}$ of the spectra.

Shultz et al. (2018) suggested that its magnetic field shows no variability over a timescale of about 35 years. In the following, we report on our results of the magnetic field measurements in all six stars.

\section{Observations and magnetic field analysis}

The observations of the He-rich early B-type stars used here were obtained on 2015 March 10, 12, and 14, 2015 June 5, and 2015 October 20 using the High Accuracy Radial velocity Planet Searcher in polarimetric mode (HARPSpol; Snik et al. 2011) installed at the ESO La Silla $3.6 \mathrm{~m}$ telescope. All spectra have a resolving power of about $R=110000$ and cover the spectral range $3780-6910 \AA$, with a small gap between $5259 \AA$ and $5337 \AA$. The reduction and calibration of these spectra was performed using the HARPS data-reduction software available on La Silla. The normalization of the spectra to the continuum level is described in detail by Hubrig et al. (2013). A summary of the HARPSpol observations is given in Table 1.

To study the presence of a magnetic field in the Bp stars, we employed the least-squares deconvolution (LSD) technique (Donati et al. 1997). This technique combines line profiles (using the assumption that line formation is similar in all lines) centred at the position of the individual lines given in the line mask and scaled according to the line strength and the sensitivity to a magnetic field (i.e. to the Landé factor). The resulting average profiles (Stokes $I$, Stokes $V$, and diagnostic null spectrum $N$ ) obtained by combining several lines, yield an increase in the signal-to-noise ratio $(\mathrm{S} / \mathrm{N})$ as the square root of the number of the lines used. For the studied stars, the line masks corresponding to $T_{\text {eff }}$ and $\log g$ determined by Zboril \& North (1999; see Sect. 3), were constructed using the Vienna Atomic Line Database (VALD; Kupka et al. 2011; Ryabchikova et al. 2015). Only lines actually visible in the observed spectra have been used to calculate the LSD profiles. Lines blended with hydrogen lines and lines in spectral regions contaminated by telluric features were excluded from the line list. The mean longitudinal magnetic field was evaluated by computing the first-order moment of the Stokes $V$ profile according to Mathys (1989).

\section{Longitudinal magnetic field measurements}

As already mentioned in Sect. 1, in He-rich stars, the magnetic field and the large-scale distribution of certain elements, in particular helium and silicon, is non-symmetric with respect to the rotation axis. The majority of studies of He-rich stars
Table 2. Magnetic field measurements for the He-rich stars using four different line masks.

\begin{tabular}{|c|c|c|c|c|}
\hline Star & $\begin{array}{c}\left\langle B_{z}\right\rangle_{\text {All }} \\
(\mathrm{G})\end{array}$ & $\begin{array}{c}\left\langle B_{z}\right\rangle_{\text {metal }} \\
(\mathrm{G})\end{array}$ & $\begin{array}{c}\left\langle B_{z}\right\rangle_{\mathrm{He}} \\
(\mathrm{G})\end{array}$ & $\begin{array}{c}\left\langle B_{z}\right\rangle_{\mathrm{Si}} \\
(\mathrm{G})\end{array}$ \\
\hline $\mathrm{CPD}$ & $-1125 \pm 29$ & $-1282 \pm 37$ & $-933 \pm 27$ & $-695 \pm 29$ \\
\hline $\mathrm{HD}$ & 1488 & 153 & 1426 & $1446 \pm 31$ \\
\hline $\mathrm{HD}$ & $370=$ & 377 & $667 \pm 30$ & $403 \pm 13$ \\
\hline HD 149257 & $-161 \pm 17$ & $-189 \pm 27$ & $-70 \pm 18$ & $-256 \pm 43$ \\
\hline $\mathrm{CPD}-$ & $466 \pm 43$ & $414 \pm 54$ & $868 \pm 44$ & $402 \pm 87$ \\
\hline HD 16 & $863 \pm 11$ & $955 \pm 15$ & $712 \pm 19$ & $675 \pm 10$ \\
\hline HD $168785^{b}$ & $1040 \pm 12$ & $1153 \pm 17$ & $1145 \pm 24$ & $915 \pm 13$ \\
\hline
\end{tabular}

Notes. The measurement for HD 168785 denoted by ${ }^{(a)}$ was taken on 2015 March 14 while the one denoted by ${ }^{(b)}$ was taken on 2015 October 10.

have revealed a kind of symmetry between the topology of the magnetic field and the element distribution (see e.g. Wade et al. 1997; Yakunin et al. 2015; Hubrig et al. 2017). Therefore, the correspondence between the magnetic field structure and the location of chemical spots can be roughly probed by measuring the longitudinal magnetic field using spectral lines of inhomogeneously distributed elements separately. As an example, the main concentration of $\mathrm{He}$ is frequently observed in the vicinity of the positive magnetic pole and Si-deficient spots around both poles. High-concentration Si spots are usually located close to the magnetic equator. We note, however, that given the availability of only single observing epochs for each target, the suggestions on the element locations in respect to the magnetic field topology should be considered very tentative.

To take the effect of the inhomogeneous surface element distribution into account, we decided to use four different line masks for the measurements, one including all photospheric lines apart from the hydrogen lines, one mask with all metal lines visible in the spectra, one mask containing exclusively He lines, and one mask containing exclusively the Si lines. The magnetic field measurements for the He-rich stars using these four different line masks are presented in Table 2 and the resulting LSD Stokes $I, V$, and diagnostic $N$ profiles are illustrated in Figs. 1-6. For all cases, the false alarm probability (FAP) is less than $10^{-5}$, confirming definite detections, and the null spectra appear flat, indicating the absence of spurious polarization. In the following, we briefly discuss the measurements of the longitudinal magnetic field using the different line masks for each star separately.

$C P D-27^{\circ} 1791$. This star was identified as helium-rich by Garrison et al. (1977). The longitudinal magnetic field in CPD $-27^{\circ} 1791$ is measured for the first time. As is shown in Fig. 1, apart from the clear Zeeman feature calculated for the line mask with the He lines, all other Zeeman features calculated for line masks containing metal lines present a distinct substructure likely related to an inhomogeneous surface element distribution. In agreement with this suggestion, the LSD Stokes I profiles calculated for these line masks appear asymmetric (best visible in the Stokes I profile calculated for the Si lines), indicating the presence of surface chemical spots. The lowest value for the longitudinal magnetic field, $\left\langle B_{z}\right\rangle=-695 \pm 29 \mathrm{G}$, is measured using the line mask containing the Si lines suggesting that we probably observe this star close to the magnetic pole and that the Si lines may form in a spot at some distance from the magnetic pole. The atmospheric parameters $T_{\text {eff }}=22700 \mathrm{~K}$ and $\log g=4.53$, typical for a spectral type B1.5-B2, were determined by Zboril \& North (1999), who used Kurucz models (Kurucz 1992). 


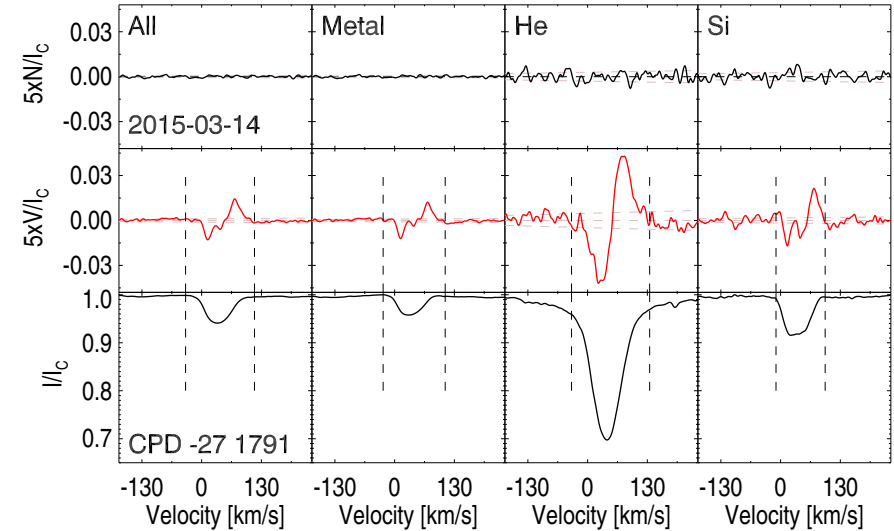

Fig. 1. LSD Stokes $I, V$, and diagnostic null $N$ profiles (from bottom to top) calculated for $\mathrm{CPD}-27^{\circ} 1791$ using a line mask containing all spectral lines, a line mask with only metal lines, a line mask containing exclusively He lines, and a line mask containing exclusively Si lines (from left to right). Vertical dashed lines indicate the integration ranges for the determination of $\left\langle B_{z}\right\rangle$.

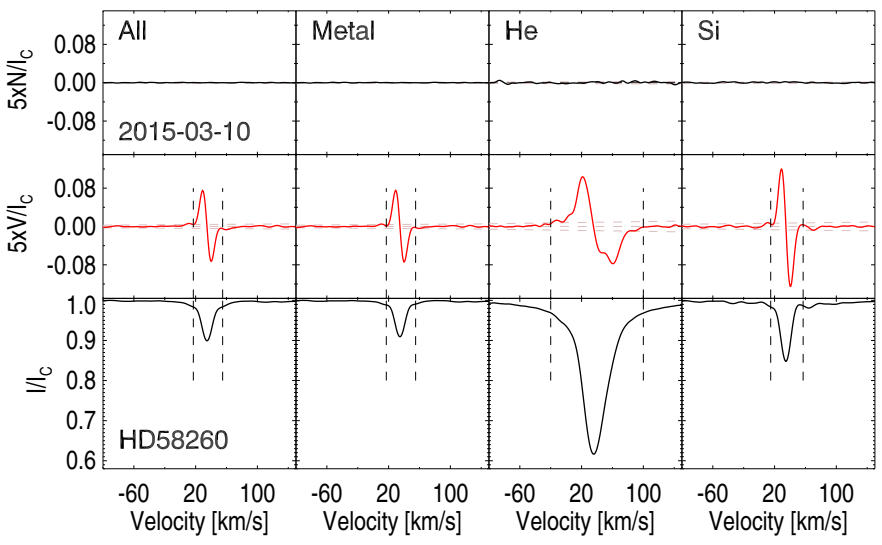

Fig. 2. As in Fig. 1, but for HD 58260.

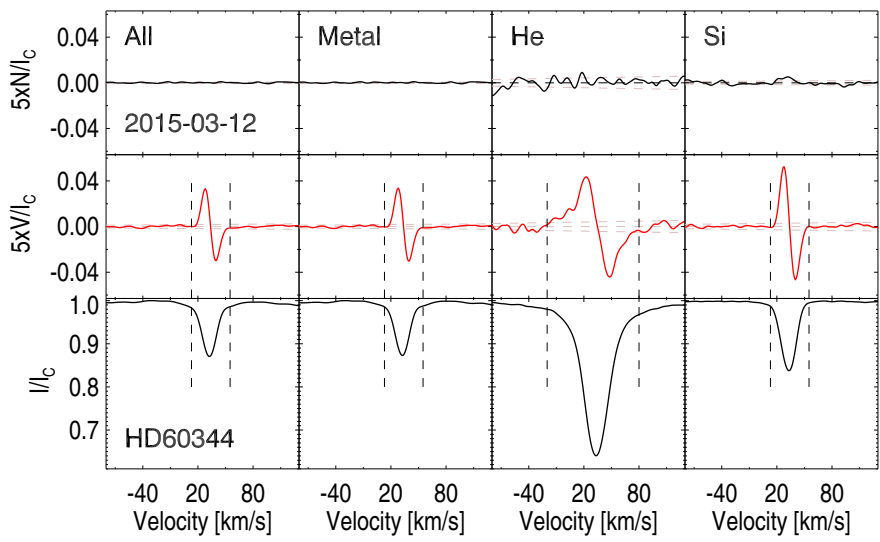

Fig. 3. As in Fig. 1, but for HD 60344.

HD 58260. This star was identified as helium-rich by Garrison et al. (1977). According to Bohlender et al. (1987), the magnetic field of this star has been constant at $2300 \mathrm{G}$ between 1977 December and 1986 March. Using these historical measurements along with nine more recent Echelle SpectroPolarimetric Device for the Observation of Stars (ESPaDOnS; Donati et al. 2003, 2006) measurements, Shultz et al. (2018)

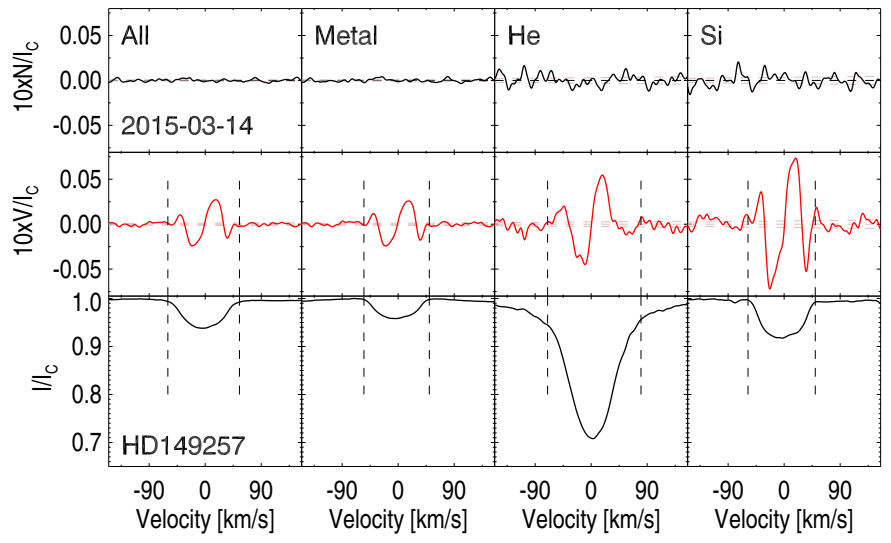

Fig. 4. As in Fig. 1, but for HD 149257.

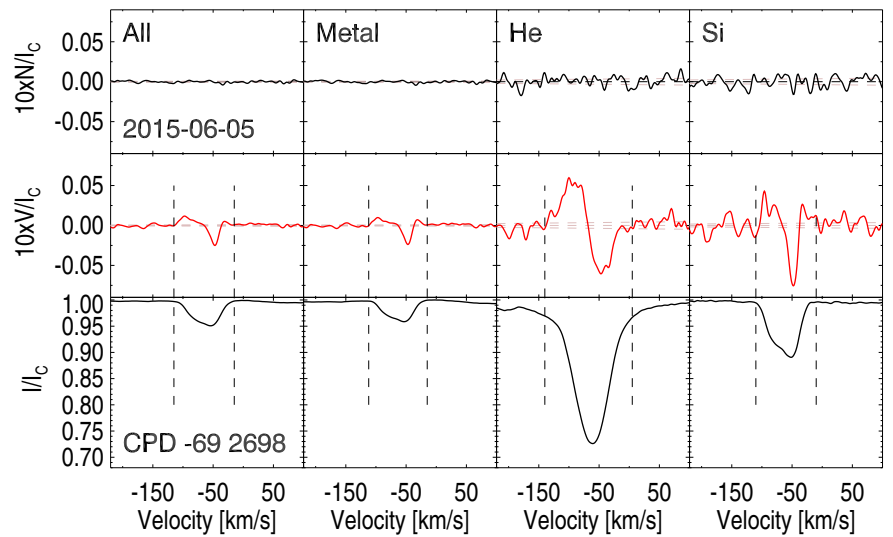

Fig. 5. As in Fig. 1, but for CPD- $69^{\circ} 2698$.

reported a $\left\langle B_{z}\right\rangle$ of the order of $1.8 \mathrm{kG}$ measured in spectra obtained in 2013-2014. The authors were unable to determine a variability period for this star due to the negligible variation of $\left\langle B_{z}\right\rangle$. All available measurements were within $1 \sigma$ of the mean ESPaDOnS $\left\langle B_{z}\right\rangle$, suggesting a non-variability of the longitudinal magnetic field over a timescale of about 35 years. Our measurements show $\left\langle B_{z}\right\rangle$ values in the range $1430-1540 \mathrm{G}$, and are weaker than the values reported by Shultz et al. (2018) by $\sim 300 \mathrm{G}$. To check the robustness of our result indicating a $300 \mathrm{G}$ difference between our measurements and the measurements of Shultz et al. (2018), we downloaded the ESPaDOnS spectrum of HD 58260 from the PolarBase ${ }^{1}$ (Petit et al. 2014; Donati et al. 1997). This spectrum was obtained on 2014 January 11 and is presented in Fig. 1 in the paper of Shultz et al. (2018). Using the same line list as that used in our HARPS observations we obtain $\left\langle B_{z}\right\rangle_{\text {metal }}=1882 \pm 40 \mathrm{G}$. This value is in agreement with the value $\left\langle B_{z}\right\rangle_{\text {metal }}=1820 \pm 30 \mathrm{G}$ published by Shultz et al. In Fig. 7 we present the comparison between the LSD Stokes $I$ and $V$ profiles calculated for HD 58260 using the HARPS and ESPaDOnS spectra. The strongest longitudinal magnetic field is detected using the line mask with the metal lines suggesting that we probably observe this star at some distance from the magnetic pole. The atmospheric parameters $T_{\text {eff }}=19000 \mathrm{~K}$ and $\log g=4.02$, typical for a spectral type B2.5, were determined by Zboril \& North (1999).

1 http://polarbase.irap.omp.eu 


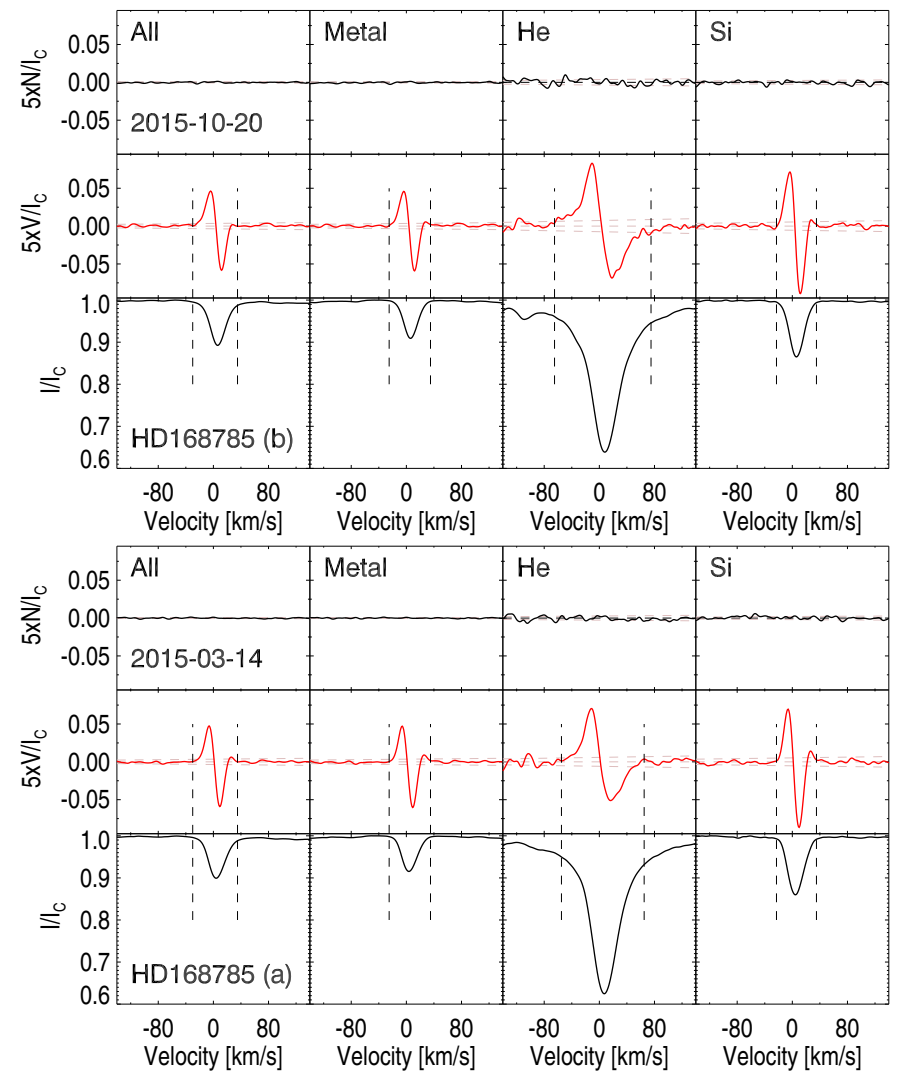

Fig. 6. As in Fig. 1, but for HD 168785.

HD 60344. The longitudinal magnetic field in this star is measured for the first time. The helium-rich nature of this star was for the first time mentioned by MacConnell et al. (1970). A longitudinal magnetic field was not detected in the previous studies by Borra \& Landstreet (1979) and Bohlender et al. (1987). Our observations clearly show evidence of the presence of a magnetic field of the order of a few hundred Gauss. The strongest longitudinal magnetic field, $\left\langle B_{z}\right\rangle=667 \pm 30 \mathrm{G}$, is measured using the He lines, indicating that the He lines may form in a spot close to the positive magnetic pole. The atmospheric parameters $T_{\text {eff }}=21700 \mathrm{~K}$ and $\log g=4.48$, typical for a spectral type B2, were determined by Zboril \& North (1999).

$H D$ 149257. The longitudinal magnetic field of this star is measured for the first time. The helium-rich nature of this star was for the first time mentioned by Garrison et al. (1977). Wiegert \& Garrison (1998) discovered significant variations in the hydrogen and helium lines. As is shown in Fig. 4, the Zeeman features calculated for all masks exhibit profiles reminiscent of a typical crossover profile and correspond to the lowest longitudinal magnetic fields measured in our star sample. Such profile shapes are usually observed when both the negative and the positive magnetic pole become visible at the time of observation, that is, we observe HD 149257 at this epoch close to the magnetic equator. The presence of a distinct substructure in the LSD Stokes I profile obtained for the mask with the silicon lines is most likely related to an inhomogeneous surface $\mathrm{Si}$ distribution. The strongest longitudinal magnetic field, $\left\langle B_{z}\right\rangle=-256 \pm 43 \mathrm{G}$, is measured using the mask with the Si lines supporting the assumption that Si spots are frequently distributed close to the magnetic equator. Bagnulo et al. (2006) observed HD 149257 in 2004 using the FOcal Reducer low dispersion Spectrograph (FORS 2; Appenzeller et al. 1998)
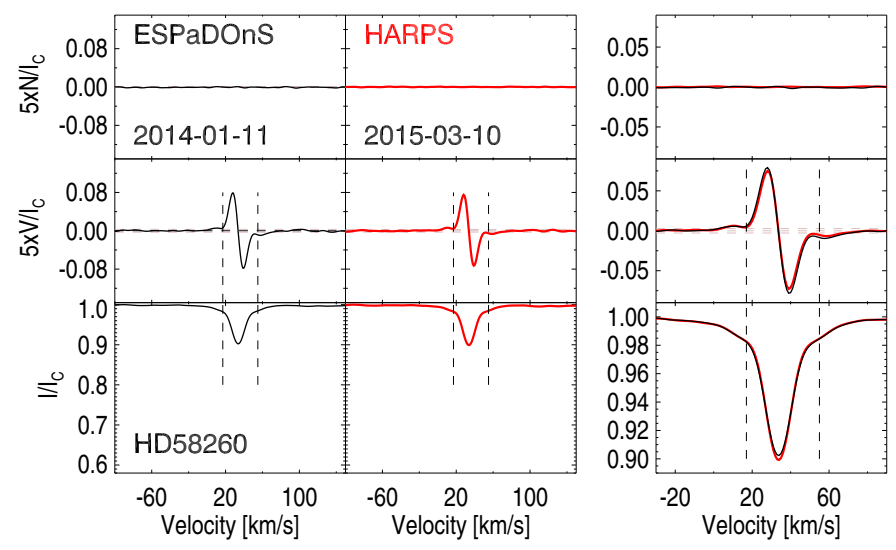

Fig. 7. LSD Stokes $I, V$, and diagnostic $N$ profiles calculated for HD 58260 using the ESPaDOnS spectrum (black line, left panel) and the HARPS spectrum (red line, middle panel). A zoom-in to the overplotted profiles is presented in right panel.

mounted on the $8 \mathrm{~m}$ Antu telescope of the VLT and did not detect a magnetic field. The atmospheric parameters $T_{\text {eff }}=24900 \mathrm{~K}$ and $\log g=4.19$, typical for a spectral type B1.5, were determined by Zboril \& North (1999).

$C P D-69^{\circ} 2698$. The longitudinal magnetic field in this star is measured for the first time. The helium-rich nature of this star was for the first time mentioned by MacConnell et al. (1970). Apart from the very clear Zeeman feature calculated for the line mask with the He lines, with a shape characteristic for a longitudinal magnetic field of positive polarity, all other Zeeman features calculated for the line masks containing metal lines exhibit a distinct substructure, probably related to an inhomogeneous surface element distribution. In agreement with this suggestion, the LSD Stokes $I$ profiles calculated for these line masks appear asymmetric. The longitudinal magnetic field measured using the He lines, $\left\langle B_{z}\right\rangle=868 \pm 44 \mathrm{G}$, is stronger by almost a factor of two than the longitudinal magnetic field measured using the metal lines. This suggests that the He lines may form in a spot that is closer to the magnetic pole, while the metal lines form at some distance from the magnetic pole. The atmospheric parameters $T_{\text {eff }}=25300 \mathrm{~K}$ and $\log g=3.90$, typical for a spectral type B1, were determined by Zboril \& North (1999).

$H D$ 168785. The helium-rich nature of this star was first mentioned by MacConnell et al. (1970). Wiegert \& Garrison (1998) discovered significant variations in the hydrogen and helium lines. The longitudinal magnetic field in this star is measured for the first time. This star was observed twice, on 2015 March 14 and on 2015 October 20. The longitudinal magnetic field measured in October is stronger than the longitudinal magnetic field measured in March by a few hundred Gauss. For both observations the lowest longitudinal magnetic field strength, $\left\langle B_{z}\right\rangle=675 \pm 10 \mathrm{G}$ and $\left\langle B_{z}\right\rangle=915 \pm 13 \mathrm{G}$, is measured using the mask with the $\mathrm{Si}$ lines suggesting that a Si abundance spot is probably clearly visible at these observing epochs and we observe this star at some distance from the magnetic pole. The atmospheric parameters $T_{\text {eff }}=23400 \mathrm{~K}$ and $\log g=4.11$, typical for a spectral type B1.5, were determined by Zboril \& North (1999).

\section{Discussion}

Our spectropolarimetric observations with HARPSpol allowed us to detect for the first time the presence of relatively strong longitudinal magnetic fields in five He-rich stars. For the sixth 
Table 3. Summary of photometric measurements and $v \sin i$ estimates.

\begin{tabular}{llclccc}
\hline \hline Star & $\begin{array}{l}\text { ASAS3 } \\
\text { interval }\end{array}$ & $\begin{array}{c}\text { No. } \\
\text { meas. }\end{array}$ & $\begin{array}{l}\text { HipPARcos } \\
\text { interval }\end{array}$ & $\begin{array}{c}\text { No. } \\
\text { meas. }\end{array}$ & $\begin{array}{c}v \sin i_{\mathrm{ZN}} \\
\left(\mathrm{km} \mathrm{s}^{-1}\right)\end{array}$ & $\begin{array}{c}v \sin i^{b} \\
\left(\mathrm{~km} \mathrm{~s}^{-1}\right)\end{array}$ \\
\hline $\mathrm{CPD}^{2} 27^{\circ} 1791$ & 2000 Nov/2009 Dec & 997 & 1990 Jan/1993 Mar & 178 & $45 \pm 4$ & $37 \pm 3$ \\
HD 58260 & 2000 Nov/2009 Dec & 781 & 1989 Nov/1993 Mar & 124 & $45 \pm 6$ & $9 \pm 1$ \\
HD 60344 & 2000 Nov/2009 Dec & 617 & $1990 \mathrm{Mar} / 1993 \mathrm{Mar}$ & 107 & $55 \pm 6$ & $10 \pm 1$ \\
$\mathrm{HD} 149257$ & 2001 Jan/2009 Oct & 722 & & & $40 \pm 4$ & $48 \pm 2$ \\
$\mathrm{CPD}-69^{\circ} 2698$ & 2001 Jan/2009 Nov & 653 & 1989 Dec/1993 Feb & 265 & $30 \pm 3$ & $26 \pm 2$ \\
$\mathrm{HD} 168785$ & 2001 Feb/2009 Nov & 996 & & & $14 \pm 2$ & $14 \pm 1$ \\
\hline
\end{tabular}

Notes. For each star in Col. 1, we list the interval and number of measurements, for ASAS3 in Cols. 2 and 3, and for HIPPARCOS in Cols. 4 and 5. Column 6 presents the $v \sin i$ values obtained by Zboril \& North (1999) and Col. 7 has the values measured on our HARPS spectra. ${ }^{(a)}$ Shultz et al. (2018) give $v \sin i=3 \pm 2 \mathrm{~km} \mathrm{~s}^{-1}$.

References. ZN = Zboril \& North (1999), ${ }^{(b)}$ this work.
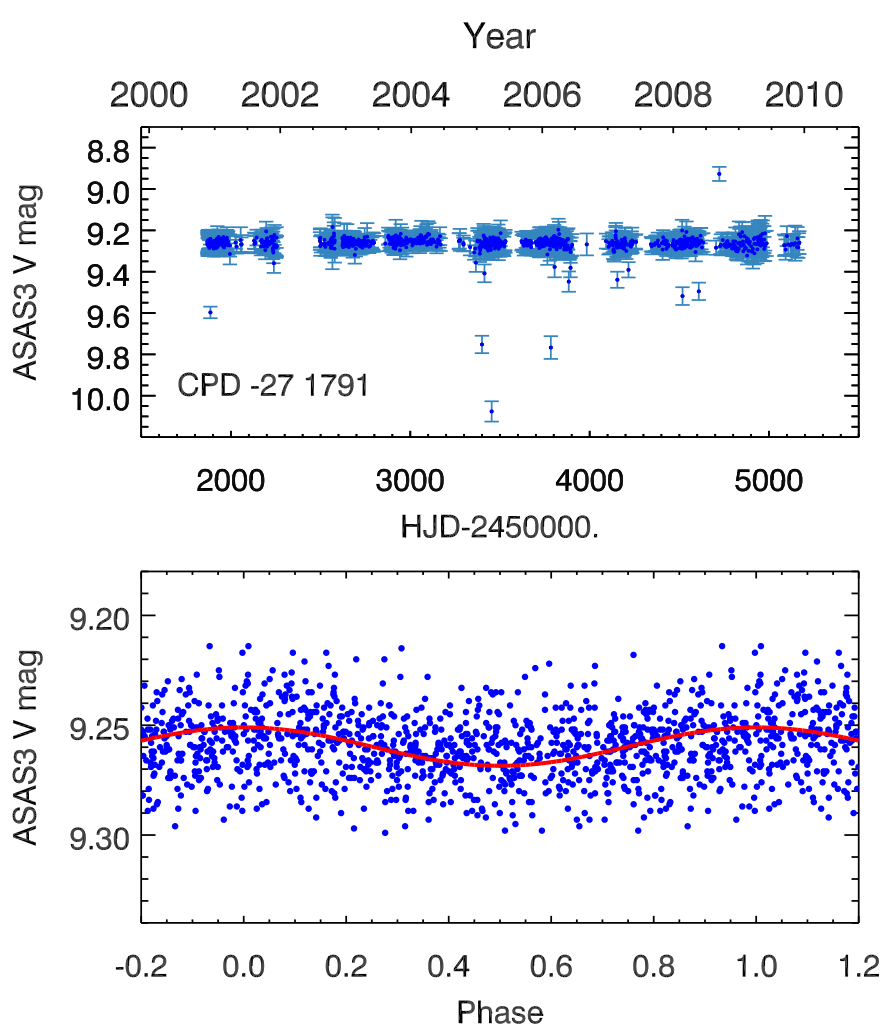

Fig. 8. Rotational period of CPD- $27^{\circ} 1791$. Top panel: ASAS3 light curve. Bottom panel: ASAS3 photometry phased with $P_{\text {rot }}=2.64119$ d.

He-rich star, HD 58260, we found an indication of a magnetic field decrease compared to previous measurements.

The measurements using different line masks belonging to different elements indicate the presence of chemical spots on the surface of all stars in our sample. Given the presence of clearly detected longitudinal magnetic fields in our targets, the determination of their magnetic periods and magnetic field geometries should be easily feasible and can be carried out in follow-up observations. Periodic magnetic variations in such chemically peculiar stars are related to the rotation periods and are generally described by the oblique rotator model (Stibbs 1950) with a magnetic field having an axis of symmetry at an angle to the rotation axis, usually called the obliquity angle $\beta$.

Since the rotation periods for our sample stars are unknown, we searched for periodic modulations using photometric data.
He-rich stars and chemically peculiar stars in general usually exhibit photometric variability due to surface chemical abundance spots. Therefore, rotational periods can be determined from photometry. We have used archival data from the ASAS3 survey $^{2}$ (Pojmanski 1997), covering the time interval from 2000 to 2009 and from the HiPParcos survey (Perryman et al. 1997; van Leeuwen 2007) covering the time interval from 1989 to 1993. The photometry time intervals and the number of measurements are summarised in Table 3 in Cols. 2-5. After removing some apparent outliers, we carried out a period search using a non-linear least-squares fit to multiple harmonics using the Levenberg-Marquardt method (Press et al. 1992). To detect the most probable period, we calculated the frequency spectrum, and for each trial frequency we performed a statistical F-test of the null hypothesis for the absence of periodicity (Seber 1977). The resulting $F$-statistics can be thought of as the total sum, including covariances of the ratio of harmonic amplitudes to their standard deviations, i.e. an S/N (e.g. Hubrig et al. 2017). Using ASAS3 observations, a single significant peak has been detected in the frequency spectrum of CPD- $27^{\circ} 1791$ corresponding to $P_{\text {rot }}=2.64119 \pm 0.00420 \mathrm{~d}$ at a high confidence level with an FAP value of $2.3 \times 10^{-9}$. No periodicity was detected in ASAS3 and in HipParcos photometry for the other five stars. In Fig. 8 we present in the upper panel the light curve collected by ASAS3 over nine years and in the lower panel the ASAS3 photometry phased with the rotation period of $2.64119 \mathrm{~d}$.

The absence of $\left\langle B_{z}\right\rangle$ phase curves (i.e. the dependence of the magnetic field strength on the rotation phase) for our sample stars does not allow us to conclude on the real field strength, which can be much higher, or the field topology. Since the determination of abundances and $v \sin i$ values depends on the chemical spot distribution and magnetic line intensification (which is different at different rotational phases), spectropolarimetric time series obtained over the rotation periods are necessary for an in-depth analysis of the atmospheres, that is, an exploration of the magnetic field strength and the surface abundance distribution would require Zeeman Doppler Imaging of the chemical abundance pattern.

Since HARPS spectra were obtained at an excellent resolving power of 110000 , we estimated projected rotational velocities for each target by fitting Gaussian profiles with full widths at half-maximum (FWHM) to the unblended $\mathrm{Ne}_{\mathrm{I}} 6402$ line and compared them with literature values. Apart from the values determined for stars HD 58260 and HD 60344, our measurements presented in Col. 7 of Table 3 appear to be in good

2 http://www.astrouw.edu.pl/asas/ 
agreement with the determinations of Zboril \& North (1999) presented in Col. 6, who used lower resolution spectra with $R=30000$ obtained with the CES spectrograph at the ESOCAT telescope on La Silla in Chile to study CNO abundances in the atmospheres of our sample stars.

The obtained results show that magnetic fields can be detected in all He-rich stars and that, in agreement with the suggestion of Osmer \& Peterson (1974), these stars form an extension of the Ap star phenomenon to higher temperatures. Similar to Ap stars, they are spectrum variables and also show variable photometrical light curves (e.g. Pedersen \& Thomsen 1977). However, while the evolution of the magnetic field strength and field geometry, including the evolution of the dipole obliquity angle $\beta$ in Ap and late-B-type stars across the main sequence, has already been the subject of several careful studies using representative stellar samples (e.g. Hubrig et al. 2000, 2007), no comparable study exists for a representative sample of early B-type He-rich stars with well-defined rotation periods and magnetic field geometries. As we showed in the previous section, the longitudinal magnetic field measurements in He-rich stars are strongly affected by the presence of chemical spots, making the determination of the obliquity angle $\beta$ difficult. Furthermore, the magnetic field geometry in these stars frequently shows contributions from non-dipolar multipoles. In particular, the distribution of the obliquity angle $\beta$ is essential to understand the physical processes taking place in these stars and the origin of their magnetic fields. As discussed by Moss (1986) and later by Hubrig et al. (2007), randomness in the $\beta$ distribution may be regarded as an argument in favour of the fossil field, since the star-to-star variations in obliquity of the magnetic field axes can plausibly be interpreted as reflecting differences in the intrinsic magnetic conditions at different formation sites. An important issue of the fossil field theory discussed in the past was the survival of the magnetic field over the star's lifetime, as it was difficult to find stable field configurations (e.g. Mestel 1984; Moss 1986). However, more recently, Braithwaite \& Spruit (2004) used three-dimensional numerical MHD simulation and showed that stable magnetic field configurations can develop through evolution from arbitrary, random initial magnetic fields. The magnetic field permeating the interstellar medium is amplified during star formation and may naturally relax into a large-scale, mostly poloidal field emerging at the surface. The work of Braithwaite \& Spruit (2004; see also the work of Braithwaite 2008, 2009; Duez et al. 2010) resulted in fundamental revision of our understanding of the behaviour of magnetic fields in stellar radiative zones. On the other hand, the magnetic field excited by a dynamo mechanism is expected to be either symmetric or antisymmetric in regard to the equatorial plane (e.g. Krause et al. 1976).

Acknowledgements. We thank the referee, G. Wade, for useful comments Based on observations made with ESO Telescopes at the La Silla Paranal Observatory under programme ID 191.D-0255. This work has made use of the VALD database, operated at Uppsala University, the Institute of Astronomy RAS in Moscow, and the University of Vienna.

\section{References}

Appenzeller, I., Fricke, K., Fürtig, W., et al. 1998, The Messenger, 94, 1 Bagnulo, S., Landstreet, J. D., Mason, E., et al. 2006, A\&A, 450, 777 Bohlender, D. A., 1988, PhD Thesis, The University of Western Ontario, Canada Bohlender, D. A., Landstreet, J. D., Brown, D. N., \& Thompson, I. B. 1987, APJ, 323,325

Bohlender, D. A., Rice, J. B., \& Hechler, P. 2010, A\&A, 520, A44

Borra, E. F., \& Landstreet, J. D. 1979, ApJ, 228, 809

Braithwaite, J. 2008, MNRAS, 386, 1947

Braithwaite, J. 2009, MNRAS, 397, 763

Braithwaite, J., \& Spruit, H. C. 2004, Nature, 431, 819

Castro, N., Fossati, L., Hubrig, S., et al. 2017, A\&A, 597, L6

Donati, J.-F., 2003, in Solar Polarization 4, eds. J. Trujillo-Bueno, \& J. Sanchez Almeida, ASP Conf. Ser., 307, 41

Donati, J. F., Semel, M., Carter, B. D., Rees, D. E., \& Collier, A. 1997, MNRAS, 291,658

Donati, J.-F., Catala, C., Landstreet, J. D., \& Petit, P., 2006, in Solar Polarization 4, eds. R. Casini, \& B. W. Lites, ASP Conf. Ser., 358, 362

Duez, V., Braithwaite, J., \& Mathis, S. 2010, ApJ, 724, L34

Garrison, R. F., Hiltner, W. A., \& Schild, R. E. 1977, ApJS, 35, 111

Hubrig, S., North, P., \& Mathys, G. 2000, ApJ, 539, 352

Hubrig, S., North, P., \& Schöller, M. 2007, Astron. Nachr., 328, 475

Hubrig, S., Ilyin, I., Schöller, M., \& Lo Curto, G. 2013, Astron. Nachr., 334, 1093

Hubrig, S., Przybilla, N., Korhonen, H., et al. 2017, MNRAS, 471, 1543

Kurucz, R. L. 1992, Rev. Mex. Astron. Astrofis., 23, 45

Krause, F., \& Oetken, L., 1976, in Physics of Ap Stars, eds. W. W. Weiss, H. Jenkner, \& H. J. Wood, IAU Colloq., 32, 358

Kupka, F., Dubernet, M.-L., \& VAMDC Collaboration. 2017, Balt. Astron., 20, 503

Landstreet, J. D., \& Borra, E. F. 1978, ApJ, 224, L5

MacConnell, D. J., Frye, R. L., \& Bidelman, W. P. 1970, PASP, 82, 730

Mathys, G. 1989, Fundam. Cosmic Phys., 13, 143

Mestel, L. 1984, Astron. Nachr., 305, 301

Morel, T., Castro, N., Fossati, L., et al. 2015, in New Windows on Massive Stars, eds. G. Meynet, C. Georgy, J. Groh, \& P. Stee, IAU Symp., 307, 342

Moss, D. 1986, Phys. Rep., 140, 1

Osmer, P. S., \& Peterson, D. M. 1974, ApJ, 187, 117

Pedersen, H., \& Thomsen, B. 1977, A\&AS, 30, 11

Perryman, M. A. C., Lindegren, L., Kovalevsky, J., et al. 1997, A\&A, 323, L49

Petit, P., Louge, T., \& Théado, S. 2014, PASP, 126, 469

Pojmanski, G. 1997, Acta Astron., 47, 467

Press, W. H., Teukolsky, S. A., Vetterling, W. T., \& Flannery, B. P. 1992, Numerical Recipes in FORTRAN, The Art of Scientific Computing (Cambridge, UK: Cambridge University Press)

Ryabchikova, T., Piskunov, N., Kurucz, R. L., et al. 2015, Phys. Scr., 90, 054005

Schöller, M., Hubrig, S., \& Fossati, L. 2017, A\&A, 599, A66

Seber, G. A. F. 1977, Linear Regression Analysis (NewYork: Wiley)

Shultz, M. E., Wade, G. A., Rivinius, T., et al. 2018, MNRAS, 475, 5144

Snik, F., Kochukhov, O., Piskunov, N., et al. 2011, in Solar Polarization 6, eds.

J. R. Kuhn, D. M. Harrington, H. Lin, et al., ASP Conf. Ser., 437, 237

Stibbs, D. W. N. 1950, MNRAS, 110, 395

van Leeuwen, F. 2007, A\&A, 474, 653

Wade, G. A., Bohlender, D. A., Brown, D. N., et al. 1997, A\&A, 320, 172

Wade, G. A., Neiner, C., Alecian, E., et al. 2016, MNRAS, 456, 2

Wiegert, P., \& Garrison, R. F. 1998, JRASC, 92, 134

Yakunin, I., Wade, G., Bohlender, D., et al. 2015, MNRAS, 447, 1418

Zboril, M., \& North, P. 1999, A\&A, 345, 244 